\title{
Attitude and Behavior towards a Scientific Exhibition on Fusion Energy: A Pilot Study of Thermographic Analysis in Psychosocial Research
}

\author{
by Sonia Ferri Anglada* and José Manuel Cornejo Álvarez
}

\author{
*CIEMAT, Ministry of Science and Innovation. CISOT, Gran Via de les Corts \\ Catalanes, 604, 08007 Barcelona, sonia.ferri@ciemat.es \\ ${ }^{* *}$ Dept. of Social Psychology, University of Barcelona. Passeig Vall d'Hebron, 171, \\ 08035 Barcelona, jcornejo@ub.edu
}

\begin{abstract}
This research intends to explore the potential applications and measurements of thermography in the investigation of human psychological and social processes. We present a pilot study that analyzes the thermal correlates associated with attitudes and behaviors of visitors to an exhibition on fusion energy. Two sequences with a total 26 image captures in this natural setting were analyzed. Results show temperature changes associated with interest in the exhibition and proactive participation. Finally, we discuss the advantages of non-intrusive techniques as demonstrated in previous literature, and we coin the 'Social Thermography' term for future research.
\end{abstract}

\section{Introduction}

This report ${ }^{1}$ is organized as follows. This first section introduces our study in a natural setting and provides a general overview of thermography, highlighting some of the straddling studies from biomedical, engineering and psychological sciences. In the second section we detail the design method and main aspects: instruments, procedure and data analysis. In the third section we describe some preliminary results of our pilot study. We focus on two case studies that highlight certain temperature trends related to visitor attitudes and behaviors throughout the tour. In the final section some of the trends are interpreted in the light of previous studies; theoretical, methodological and practical implementation of thermographic measurements in future investigations of human behavior in general and social behavior in particular is also discussed.

The aim of this study has been to explore the potential relationships between body temperature changes and attitudes and human behaviors in natural environments. This objective follows some studies in previous literature, beginning with the chemical clock hypothesis that implies an occasional link between body temperature and the perception of duration [1], through to recent contributions linking infrared thermographic measurements, mental workload and human performance [2], the human learning process [3] and driving performance [4]. This methodological and technological approach can be very useful, as shown in biomedical sciences when developing, for example, sexual arousal diagnoses [5]. Viability for this type of analysis is highly related to thermal ubiquity (similar to the case with acoustic noise ubiquity), notwithstanding the complex relationships at the physiological level (physical and psychological activity with body surface temperature regulation); it is further tied to the benefits inherent to the availability of a non-invasive tool (most important for capturing human dynamics in an environmentally friendly way). In this context, our interest focuses on exploring thermal behavior, not only of isolated individuals but also of different groups within the social interaction. Ultimately, our interest is to detect potential thermal behavior patterns related to specific power, influence, communication, learning and performance phenomena, etc.

While reviewing previous literature we found that psychological studies are not applying this technology yet, whereas other disciplines are incorporating it, which produce relevant data for possible thermographic analysis interpretations, even providing useful findings to psychology itself. Previous works include studies performed both in the civilian and the military fields, mostly with individualistic approaches, although some are group-focused. Most are linked to cognitive processes and mental workload in relation to performance gains, while others focus on some emotional process related components, linked to fatigue or stress, for example.

Hancock's study [1] is a historical reference of experimental psychology testing the chemical clock hypothesis; this hypothesis implies a causal relationship between body temperature and the subjective perception of time duration. The study shows the importance of endogenous and exogenous controls on the subjective perception of time, whereas when individuals act within social groups, external references become prominent. Experimental manipulation of the heat

\footnotetext{
${ }^{1}$ The empirical study project is part of a research project carried out by Ciemat-CISOT and the University of Cardiff within the EFDA Workprogramme 2011: Awareness \& Communication and Integrated Framework (CfP-WP11-SER-02); it is also part of WP11-SERACIF-2: Fusion Expo and its Role in Promoting Fusion Understanding. The views expressed in this presentation do not necessarily reflect those of the European Commission.
} 
temperature and the data support an endogenous function of time estimates of the thermal value thresholds. $\mathrm{A} 1^{\circ} \mathrm{C}$ temperature increase can be associated to a $10 \%$ increase in the speed estimate. Data suggest a relative insensitivity in the middle ranges of body temperature variations. Strong interaction between external information and some central processing mechanisms of perception that are thermally sensitive within certain thresholds is also suggested.

According to Almirall [6], body temperature is a particular case of a biological phenomenon usually caused by opposing forces, regardless of the timescale. This means that body temperature can be modeled throughout the day by a two-term function with potential and exponential terms. Besides, circadian rhythms also model temperature according to endogenous and exogenous components. This means that either between different days or depending on certain times of the day, processes may favor heat gain or heat dissipation.

From a biomedical approach, Kukkonen et al. [5] introduce thermography as a non-intrusive technique to relate sexual arousal in men and women. They made the hypothesis that thermal measurements differ for different induced sexual arousal conditions and neutral, positive or negative mood conditions. The study concludes that thermal imaging is a promising tool for this type of evaluation, as thermography discriminates between neutral and extreme conditions.

In this line of biomedical science studies, Diakides et al. [7] summarize some physiological mechanisms of skin blood flow and factors that influence changes in heat, such as skin temperature in response to stress. Infrared thermography, while providing accurate and objective skin temperature measurements on specific locations to capture thermal maps for regions of interest $(\mathrm{ROI})$, should be combined with other techniques.

Pavlidis [8] found that high levels of stress are associated with high periorbital perfusions and rising temperatures. Consolidating these findings, Tsiamyrtzis et al. [9] highlight the correlation between increased blood perfusion in the orbital muscles and human stress levels; these changes can be detected and quantified using thermal imaging. Specifically, observations of actual criminal interrogations show that thermographic imaging allows recognizing stress signal patterns with an $87.2 \%$ success rate on 39 subjects. The subjects show changes during critical moments of the interview, as compared to other times on the baseline.

Other studies also provided objective measurements relating temperature and stress, while incorporating and combining psychological domains and subjective assessments from industrial contexts. The study of Genno et al. [10] is a good example as it explores into the reactions of a group of operators in a nuclear plant and proposes a method to assess facial temperature sensations related to stress and fatigue. Nasal temperature measurements suggest different ways of inferring both dependent variables on the basis of assuming that fatigue is generated by accumulation of stress and that it varies among individuals. It was used on tracking tasks when evaluating image preferences with the aim of assessing its usefulness in preventing these negative states. The inference results of both states correlated significantly with the subjectively attributed values; such is the case, for example, for fatigue $(r=0.93)$. There is a relationship between nasal temperature and the temperature on the rest of the face with closed eyes.

Genno et al. [10] suggest that autonomic nervous system response and body temperature are primarily related to the necessary and sufficient amount of time devoted to training in new tasks. Learning requires a certain level of cognitive arousal, similar to that associated with other concepts such as attention or motivation. Feelings of stress or fatigue can lead to measurable levels of change in a person's body temperature, detectable by thermal imaging.

The study by Kang et al. [3] on the training of new skills in virtual situations in the military field shows the correlation between changes in facial temperature and human learning processes. Non-invasive image measurements are used to detect changes in nasal temperature during the execution of alphanumeric tasks. In general, the subjective perception of mental load decreased when nose temperature and accuracy increased. The results showed strong correlations between each of the dependent variables (temperature, $\mathrm{MCH}$ ratings). In short, they conclude that thermography can be meaningfully used in order to define appropriate training timings and effectiveness for new tasks.

The study of Or et al. [11] considers non-intrusive mental load physiological measurements through changes in facial temperature to predict performance in driving simulator tests. It demonstrates the potential of using these parameters as objective indices to infer mental workload. A significant correlation between nasal temperature changes and subjective overload scores was also identified.

Kang [2] proposes the use of the objective, effective and non-invasive means of thermographic measurement in combination with other techniques to evaluate mental workload and human performance. It does so by taking thermal images of the face in order to quantify learning and mental workload levels during alphanumeric task performance implemented in a driving simulator. Through various thermographic studies, the ability to identify sufficient training time for both simple and complex cognitive tasks is demonstrated.

Reyes et al. [4] state that physiological measurements are a welcome addition to performance and subjective measurements when assessing affective responses of vehicle drivers; this looks into the man-machine relationship, which does not require open, explicit or voluntary answers from the driver. They explore into how facial temperature may 
reflect driver response to interaction demands with in-vehicle systems. Temperature measurements suggest that drivers are sensitive to various cognitive processes that are explicitly evaluated with direction change or lane change measurements.

Although facial temperature measurements are not usually used to address driver response, they could provide a more complete profile of such response. Affective or emotional responses are often ignored; physiological measurements can additionally contribute to the alternative measurements. This study illustrates how facial temperature may reflect the demands and the affective response that drivers experience when interacting with a simulator, the Human-Machine Interface (HMI). Facial temperature is an indirect indicator of cognitive status [9].

\section{Method}

This research is an exploratory study on how facial and hand temperature can reflect individual response to the dynamics related to two groups of visitors touring a scientific exhibition on Fusion Energy. The pilot study was carried out in May, 2010. The thermographic records were registered with a professional digital infrared camera (model FLIR T335 by FLIR SYSTEMS). In all cases express consent was requested prior to capturing any images. Image capture was dynamic, following the natural sequence of the visit tour itinerary. Photographs were classified according to four variables: time sequence (during and after the guided visit), object based stimulus ${ }^{2}$ (Plasma Ball, Iter Scale Model, Reactor Site Scale Model, Text Panels, Bicycle, Assessment Phase), associated actions (listening, looking, paying attention, touching, pedaling, answering, etc.) and participation reactions (active or passive). The thermographic analysis of thermal human behavior measurements can help in discriminating and interpreting the reactions to different scenarios of demand and of interaction with the displayed elements and proposed activities and tasks during the guided visit to the exhibition. For the analysis, cases have been selected from both groups; these groups, which differed in size and participant gender and age, occurred spontaneously depending on visitor attendance. In all cases the selected thermographic photographs reflect attitudes and behaviors of the dynamics in both groups.

\subsection{Design}

We conducted an exploratory study comparing facial and hand temperature thermographs throughout the different phases and social situations during a tour at a scientific presentation on fusion energy. Among over 500 collected thermographic photographs, 26 were selected to illustrate the evolution of two independent spontaneous visitor groups, with $N=17$. The first group consisted of 6 subjects, the reference subject being an adult male aged 62 . The second group consisted of 11 subjects, the reference subject being an adult female aged 44 . Both groups made the same day visit during the morning, in two consecutive sessions: the first at 11:00 am and the second at 12:00 am.

The selection criterion was to have an equivalent sequence in both photograph series. These cases are relatively homogeneous for some of the criteria. Both came as part of a family of three, on the same day (on a weekend) and, in particular, both participated actively in some phases during and after the tour (these were, respectively, making the bicycle exercise and completing a visit assessment protocol in which, among other aspects, attitudes and satisfaction were evaluated).

However, it is clear that as a design constraint we had a variety of frames, distances and visibility for photographic composition, in addition to unequal tour lengths and time windows, all having an effect on environmental temperature averages. Nevertheless, it was possible to neutralize some of these limitations.

\section{2 Instruments}

Infrared imaging technology raises the need to consider variables such as emissivity (the difference between a blackbody and a graybody) and environmental conditions (measuring objects through different layers, air humidity and ambient temperature [12]). The FLIR T335 camera settings take into account human skin emissivity (ranging between 0.95 and 0.98$)$, reflected temperature, relative humidity and distance to the source $(2.5 \mathrm{~m}-3 \mathrm{~m})$.

[7] highlights some key issues, such as: the importance of dynamic range, meaning that the camera must preserve the fine temperature details even when taking a large thermal scene; sensitivity, measured in degrees Celsius; resolution, in relation to colors and color gradients; and temperature calibration (two methods of calibration are available: internal and external). In our case calibration was internal, performed before use, being the preferred option because it simplifies the data collection process.

\footnotetext{
${ }^{2}$ These objects, scale models and information panels, were the main items in the Fusion Expo exhibition. After the guided tour, various activities were proposed to the visitors.
} 


\subsection{Procedure}

Conditions for image capturing involved the researcher monitoring both groups during separate sessions and following the natural development of the guided tour. Each image was therefore unique, as it was captured at a specific moment and in a particular environment. Likewise, each collection was done in manual mode and generated simultaneously two types of image, infrared and digital.

\subsection{Data Analysis}

Analysis was made on each picture independently by two analysts; subsequently, final results were jointly agreed upon; the analysis was made under serial concordance criteria so as to improve data comparison reliability. Although this paper only presents thermographic images, the set of images obtained in this pilot study combine simultaneously acquired digital formats and thermographs. The program used to analyze thermographic data was FLIR QuickReport 1.2 SP1 (2009) for Windows XP. To eliminate the general effects of thermograph frame differences and ambient temperature, data matrix temperatures have been converted to relative temperatures by subtracting the overall average of each shot. We thus obtain relative temperature values that allow comparisons to be made between different captures.

Wang et al. [13] propose a new method for measuring facial temperature that combines visible and infrared facial images. Feature points and regions of interest (ROI) are located in the infrared image using artificial markers or special vision systems. This method does not require special markers while its robustness is ensured; reliable facial features such as eye or lip corners are provided by using 3D positions as control points; the method may be used for both types of images.

\section{Results}

Results show the thermal variations across tasks for museum visitors. Table 1 shows the relative temperatures for the two cases, a male visitor and a female visitor. The total sample selected consists of 26 thermographic photographs, each consisting of a sequential 13 capture selection. Figure 1 shows the differential relative temperatures in degrees Celsius $\left({ }^{\circ} \mathrm{C}\right)$ between the environmental average temperature and the direct temperature of the upper extremities for the two selected cases. The $x$-axis shows photograph sequential numbers for every case (male and female); the $y$-axis defines differential relative temperature values for every capture (face and hands). Some extreme differential values, both maximum and minimum, have been highlighted in "warm" and "cold" colors. Four pictures illustrate these extreme differential values (refer to figures 6 through 9). The Differential Relative Maximum Temperature of the Face (DRMATF) (figure $6\left[10.7^{\circ} \mathrm{C}\right]$; figure $8\left[7.8^{\circ} \mathrm{C}\right]$ ) relates to tasks involving enthusiasm and motivation, linked to the fact of playing an active role (i.e., demonstrating or experimenting) (attitudes and behaviors that link themselves to dynamic elements in order to generate energy); this is related to participation and commitment. The Differential Relative Minimal Temperature of the Face (DRMITF) (figure $7\left[8.6^{\circ} \mathrm{C}\right]$; figure $9\left[6.9^{\circ} \mathrm{C}\right]$ ) relates to tasks where "easiness" and boredom become evident, as associated to a passive role (i.e., observation or introspection) (attitudes and behaviors that link themselves to large scale models containing more static than dynamic elements); this is related to lower participation and commitment, but not always.

Table 2 shows average relative temperatures and face and hand correlates for the two visitor cases. The average temperature between head and hands is about $1.22^{\circ} \mathrm{C}$ for the whole of the 26 infrared photographs. With respect to these $1.22^{\circ} \mathrm{C}$, temperature decreases by $0.32^{\circ} \mathrm{C}$ in the male thermogram series while the value increases by $0.33^{\circ} \mathrm{C}$ for the female series. A general trend shows that hand temperatures are about $1.22^{\circ} \mathrm{C}$ below head temperatures. This difference is greater in the female subject $\left(1.55^{\circ} \mathrm{C}\right)$ than in the male subject $\left(0.90^{\circ} \mathrm{C}\right)$. Furthermore, average head temperature is higher in the male subject $\left(9.18^{\circ} \mathrm{C}\right)$ than in the female subject $\left(7.37^{\circ} \mathrm{C}\right)$. This difference is higher for hand temperatures, $8.28{ }^{\circ} \mathrm{C}$ for the male visitor and $5.82^{\circ} \mathrm{C}$ for the female visitor. Pearson's linear correlation coefficient between face and hands for this thermogram set is 0.95 . These results could be explained in part by the time difference (later in the day for the female subject), due to the higher environmental temperature (between 11:00 $\mathrm{h}$ and 12:00 $\mathrm{h}$ ). Figures 2, 3 and 4 show table results.

Figure 2 shows a graph plotting face and hand correlations for all the male series thermographs $(r=0.96)$. Figure 3 shows a graph plotting face and hand correlations for all the female series thermographs $(r=0.71)$. It is noticeable that in this case temperatures tend to be at a significantly lower level and more concentrated. Figure 4 shows a graph plotting face and hand correlations for all thermographs in both series $(r=0.95)$. The explanation for the apparent discrepancy of the joint correlation for both series, when compared to each separate correlation, is that sets are complementary (higher temperature positions for the male visitor and lower temperature positions for the female visitor). These results show differences consistent with those found in other studies and support the overall quality of the performed thermography, especially when considering that approaches and positions have changed throughout the captures.

Table 3 summarizes temperature differences between the male subject and the female subject, both for face and hands, grouping thermographs according to three descriptive variables: topic (Plasma Ball, Iter Scale Model, Reactor Site Scale Model, Text Panels, Bicycle and Assessment Phase), moment in time (during or after exhibition) and degree of 
participation (active or passive). Hand temperature basically follows the head temperature pattern, with approximately a $-1{ }^{\circ} \mathrm{C}$ difference. Results show temperature characteristic variations between series for the various analyzed variables and, particularly, the opposite directions taken by sequential variations in participation. Figure 5 shows the results of this table.

Figure 5 shows the similarity of face and hand temperature variation profiles between both series for the three descriptive variables.

Regarding the first variable, we observe the difference in the focus of attention as created by the various topics. For the male subject, the most valued topic is the Bicycle, according to both face and hand temperatures $\left(10.65^{\circ} \mathrm{C}\right.$ and $9.95{ }^{\circ} \mathrm{C}$ respectively), while the least valued topic is Assessment Phase $\left(6.90^{\circ} \mathrm{C}\right.$ and $\left.5.26{ }^{\circ} \mathrm{C}\right)$. For the female subject, different topics have an homogeneous corresponding temperature around $7.6^{\circ} \mathrm{C}$. However, there is a decline to $4.53^{\circ} \mathrm{C}$ in hand temperature on Iter scale model thermographs.

In the second variable we also notice a difference between male and female subjects. For the male subject, face temperature during the exhibition is of $9.59^{\circ} \mathrm{C}$ and decreases to $6.90^{\circ} \mathrm{C}$ after the visit. For the female subject, the situation shows the reverse. During the exhibition, face temperature is of $7.27^{\circ} \mathrm{C}$ and increases to $7.60{ }^{\circ} \mathrm{C}$ after the visit. This effect is magnified in hand temperatures $\left(5.42^{\circ} \mathrm{C}\right.$ and $6.73^{\circ} \mathrm{C}$ respectively). This temperature increase coincides with the active behavior of touching the Plasma Ball as an attitude and gesture that expresses particular pleasure and the closing of that family visit.

Again, the third variable reflects an inverse attitude and behavior between the two series. In this case and for face captures, active participation of the male visitor (e.g., cycling or filling in a questionnaire in the assessment phase) took place at a lower temperature $\left(8.78^{\circ} \mathrm{C}\right)$ than passive participation (e.g., listening, watching), which show higher values $\left(9.36^{\circ} \mathrm{C}\right)$. The opposite is true for the female visitor $\left(7.58{ }^{\circ} \mathrm{C}\right.$ and $7.24^{\circ} \mathrm{C}$ respectively). This trend is stronger for hand temperatures.

Overall, these results show the sensitivity of thermography when applied to social situations, in addition to its discriminative capabilities. Temperature variations appear to account for the different psychophysical and psychosocial processes present during the tour.

\section{Discussion and Conclusions}

These preliminary results, in sum, show that the maximum differentials in facial temperatures seem to be related to attitudes of involvement and active behaviors, as a form of appropriation, and to active and procedural learning or vicarious learning by means of the observation of others [14]. The minimum differentials in facial temperatures seem to be related to situations of absorption and concentration that require activating the cognitive component and that occasionally may link with diminishment of attention, fatigue or lack of interest. These results can relate respectively to some findings of Environmental Psychology in Museums and visitor behavior [15] [16]. The effects of innovation, large size objects, mobile components, pleasant aesthetics, sensorial and interactive factors with other variables that positively modulate attention, a positive attitude of interest; these facilitate certain behaviors such as the emissivity in the psychomotor responses and procedural learning. The effects of monotony, small size objects, static components, poor aesthetics, sensorial and interactive factors with other variables that negatively modulate attention, lack of interest; these hinder the emissivity in the psychomotor responses and procedural learning.

The pilot study and the above preliminary findings encourage us to further apply thermography to different human and social interaction situations, addressed by Differential Psychology or Social Psychology amongst others. Certainly it will be necessary to perform numerous experiments with controlled designs. We believe that this methodological strategy can contribute valuable information on psychosocial phenomena that sometimes is difficult to evaluate in real time. Additionally, it provides a useful complement both to systematic observation technologies and to other types of subjective evaluations of the affective, cognitive and/or behavioral domains, in order to assist in clarifying inaccessible aspects of other evaluation perspectives during future research.

In the interpretation of thermal images captured in social settings, specific references to environmental contexts are found, such as those contributed in other studies [15] [16] [17]. Bitgood [17] defines that visitor attention as a psychological and physiological response acts as a guide when providing group training; three basic stages are involved: attention attracted, focus and engagement. This becomes motivated by the interaction of personal factors related to interests and values and of environmental factors related to the exhibition facilities. Intellectual involvement is often accompanied by emotional excitement and may be enhanced when physical interaction occurs. A large number of cognitive processes require attention and strong engagement; these include learning, flow, immersion, restoration of attention, etc.

It is assumed that the processes described in cognitive and affective social psychology provide the best results for what happens with visitors in exhibition spaces. It is assumed that physical and mental states have a significant influence on 
attention and fatigue (whether physical or mental), and may diminish attention. Fatigue may be associated with stress, lack of sleep or prolonged physical or mental exercise. Furthermore, energy levels may vary from one day to another depending on stress, physical condition, etc. When energy levels are low, the effort of reading lengthy labels or walking long distances is less likely to be accepted. There is an aspect that has been already noticed by many authors, which to us is particularly interesting; it is the strong influence of group members or of the presence of other visitors on the individual's dynamics while visiting the exhibition. Group members can significantly influence the type of display that is selected or the level of attention and commitment.

The usefulness of thermography in biological applications has been demonstrated by [12], who stressed the advantages related to speed and ease in attracting mobile targets: procedures do not require contact with non-accessible targets, there are no interferences, targets are not required to spend energy, there is no risk of contamination, and no mechanical effects are produced on objects. Similarly, the advantages of thermographic applications in measuring various psychological domains are multiple. Thermographic evaluation is non-intrusive; this facilitates both subject participation (it is comfortable because it does not require any effort on their part, and natural as it does not involve a change of spontaneous dynamics) and researcher rigor (provides reliability as data related to thermal reactions are difficult to distort or falsify). Another advantage is real time data collection and assessment; this feature makes it easier to detect any variation and relate it to stimuli, conditions or critical situations.

Additionally, thermographic measurements, as the case was once with physiological measurements, are a good complement to objective measurements (e.g., performance measurements) and subjective measurements (e.g., psychological states). In fact, the latter (subjective measurements) can lead to a loss or alteration of information because of their intrusiveness. Our pilot study represents a good example for a case study in which, through the metrics of upper extremity temperatures (head and hands), the presence or concurrence of both external and internal processes relating to individuals and groups is to be inferred. In short, it aims at capturing traces of psychological and psychosocial processes in situations of demand and human interaction.

Our pilot study has some limitations, so we wish to emphasize the need of applying care when reading this information, both regarding the preliminary results and the initial derived conclusions. Ultimately, results should be considered as trends, until future studies and greater depth analyses are carried out.

We are bound to recognize methodological and practical limitations in this study that affect the reading of the preliminary results presented. There is a weakness in the method tied to case studies and the lack of sample representativeness; two small groups and individual cases cannot be extrapolated to other populations, therefore conclusions are bound in time to the particular cases. Furthermore, although several thermographic data were obtained for each case, it becomes difficult to work with inter-individual differences. A thermographic data baseline was not available either, this precluding comparisons with thermal values under other conditions (e.g., pre-test and post-test). Moreover, the analysis becomes difficult due to missing data caused by the dynamic motion of the group; it was not always possible to obtain all the data for each case (for example, those cases where there are overlaps in a 2D image: part of the image of a subject is hidden by the superimposed image of another subject). Another aspect relates to improved design and better control of image capture distances. Possible solutions that occur to us would be placing several cameras at fixed points along the tour or using telemeters to assess frame object distances in real time. Besides this technical handicap, another effect we have also tried to minimize is the impact of systematic observation, typically associated to the direct observation by the researcher following the group. This would be an aspect to improve on; it could be explored in future research work in order to define the impact of cameras on human behavior, as demonstrated in other studies [15].

Note that here it is not very easy to identify facial areas and specific locations (such as nose, forehead, cheeks, neck), as opposed to the case with individual psychology, where access to these specific points is much easier and less data can be missed. Measurement accuracy and database completeness become a harder issue. In this sense we agree with Wang et al. [13]; it was difficult to extract features in infrared images, even salient facial features, due to a lack of resolution or excessive noise, to the need for developing specific programs to solve calibration problems, and to resolution issues when superimposing infrared and digital images.

Kukkonen et al. [5] indicate that equipment cost is a limitation for thermography, together with the lack of a standardized method to examine data and assess the difficulties in securing it in the regions of interest (ROI); here the differences relate to different thermal areas even when locations are very close (in the $\mathrm{mm}$ range), and to male and female temperature differences; other variations are related to external factors or menstrual cycles in the female case. Furthermore, the importance of biological rhythms and the plasticity of temperature control throughout the day have also been demonstrated [6].

Thermography, applied to a wide range of natural settings, as well as information technology systems such as Telemetric Monitoring [18], can be useful for behavioral sciences since they have the potential to detect processes or characteristic patterns of change over time and to evaluate the effects of certain interventions. Quantitative measurements may show a wide range of behaviors and events in natural settings. Telemetrics, like Thermography, include passive and active technologies. 
Our review of the international background has highlighted some relevant studies. However, it was to our regret that we did not find national contributions to this subject; nevertheless, our initial expectations aim at opening, extending and consolidating future thermographic applications in such an undeveloped field where references are clearly scarce. This finding seems paradoxical from the standpoint of the benefits that may be obtained from deploying non-intrusive techniques and technologies that have proven so useful in both psychosocial observation and experimentation, showing to be particularly valuable in assessing real-situation attitudes and behaviors.

Cognitive-emotional processes such as behavioral changes occur with changes in temperature, whether it is an individual or a social situation. Situations of social interaction especially stimulate the generation and change of attitudes. Potentially, these can be captured by thermography to describe the different roles generated; it is true, however, that the study of attitudinal processes is complex and often it becomes difficult to separate cognitive and affective components.

MacLin et al. [19] suggest the 'Social Psychophysics' term to apply the methods of psychophysics and respond to social issues by providing a new dimension of experimental manipulation and control of the presentation of stimuli and response capture. However, they recognize that from this perspective, relevant stimuli in social psychology tend to be complex and difficult to quantify; control problems are also highlighted in their study. In a similar way, we propose the 'Social Thermography' term to refer to the application of thermography to general interaction and social influence issues.

Thermographic analysis of social settings can provide a thermal hierarchy that could potentially uncover the underlying psychological and psychosocial processes. Energy flows and is distributed non-randomly. The thermal image is an energy component of the captured social situation when interactions take place. The thermographic element is an objective correlative that could potentially capture energy exchanges between individuals co-present at certain defined times and scenarios. Thermographic interpretation necessarily requires their integration into the broader situational context, which provides content and specificity.

We believe in potential projections of 'Social Thermography' applied to human groups. In addition to the proven benefits of its non-intrusiveness, it could bring significant elements of triangulation for multiple-assessment observations, including the advantages of mixed methods [20] [21].

In addition to new research, our purpose in the future would include a review, through thermography, of some main results of current psychosocial research work. With the assistance of thermography, some classical concepts such as climate or social status, influence and roles, etc., may be contrasted against new measurements and meaningfully reconceptualized into unified terms.

\section{Acknowledgments}

The authors would like to thank the European Fusion Development Agreement (EFDA Workprogramme 2011), CIEMAT, the Socio-Technical Research Center (CISOT), the Socio-Technical Research Laboratory, Cardiff University, University of Barcelona and the Department of Social Psychology for their special support; thanks to the Museum of Science and Technology of Catalonia (mNACTEC) for making their facilities available. Finally, we thank the students at the Polytechnic University of Catalonia for their close collaboration, and the visitors to the museum exhibition for their participation. 


\section{REFERENCES}

[1] Hancock, P.A. (1993). Body temperature influence on time perception. The Journal of General Psychology, 120 (3), pp. 197-215.

[2] Kang, J. (2009). Effective mental workload measurement by thermography. Quantifying cognitive workload and defining training time requirements during performing simple and complex tasks. Saarbrücken, Germany. VDM Verlag Dr. Müller.

[3] Kang, J., McGinley, J.A., McFadyen, G.and K. Babski-Reeves (2006). Determining learning level and effective training times using thermography. Proceedings of 25th Army Science Conference, V43, Orlando, FL. Nov. 27-30, 2006.

[4] Reyes, M.L., Lee, J.D., Liang, Y., Hoffman, J.D. and Huang, R.W. (2009). Capturing driver response to in-vehicle human-machine interface technologies using facial thermography. Proceedings of the Fifth International Driving Symposium on Human Factors in Driver Assessment, Training and Vehicle Design, Big Sky, Montana, EEUU.

[5] Kukkonen, T.M., Binik, Y.M., Amsel, R. and Carrier, S. (2007). Thermography as a physiological measure of sexual arousal in both men and women. The Journal of Sexual Medicine, 4, pp. 93-105.

[6] Almirall, H. (1997). Modeling the body temperature throughout the day with a two-term function. Behavior Research Methods, Instruments, \& Computers, 29 (4), 595-599.

[7] Diakides, N.A. and Bronzino, J.D. (2008). Medical Infrared Imaging. New York: CRC Press Taylor \& Francis Group.

[8] Pavlidis, I. and Levine, J. (2002). Thermal image analysis for polygraph testing. IEEE Engineering in Medicine and Biology Magazine, 21 (6), 56-64.

[9] Tsiamyrtzis, P., Dowdall, J., Shastri, D., Pavlidis, I.T., Frank, M.G. and Ekman, P. (2007). Imaging facial physiology for the detection of the deceit. International Journal of Computer Vision, 71 (2), pp. 197-214.

[10] Genno, H., Ishikawa, K., Kanbara, O., Kikumoto, M., Fujiwara, Y., Suzuki, R. and Osumi, M. (1997). Using facial skin temperature to objectively evaluate sensations. International Journal of Industrial Ergonomic, 19 (2), pp. 161-171.

[11] Or, C.K.L. and Duffy, V.G. (2007). Development of a facial skin temperature-based methodology for non-intrusive mental workload measurement. Occupational Ergonomics, 7, pp. 83-94.

[12] Kastberger, G. and Stachl, R. (2003). Infrared imaging technology and biological applications. Behavior Research Methods, Instruments and Computers, 35 (3), 429-439.

[13] Wang, J. G. and Venkteswaralu, R. (2003). Pose for Fusing Infrared and Visible Spectrum Imagery. International Conference Audio-and Video-based Biometric Person Authentication (Guildford, UK, June 9-11). Jose Kitter and MIMark S.Nixon, eds. Lecture Notes in Computer Science vol. 2688, Springer, 2003. pp. 955-963.

[14] Bandura, A. and Walters, R.H. (1963). Social learning and personality development. New York. Holt, Rhinehart y Winston.

[15] Bitgood, S. (2002). Environmental psychology in museums, zoos, and other exhibition centers. In R. Bechtel \& A. Churchman (eds.). Handbook of Environmental Psychology, John Wiley \& Sons. Pp. 461-480.

[16] Patterson, D. D. and Bitgood, S. (1988). Some evolving principles of visitor behavior. In Visitor Studies 1988: Theory, Research and Practice, ed. ed. Stephen Bitgood, James T. Roper, and Arlene Benefield (Jacksonville, AL: Center for Social Design, 1988), 40-50; Dobbs and Eisner, "Silent Pedagogy in Art Museums.".

[17] Bitgood, S. (2010). An attention-value model of museum visitors.

http://caise.insci.org/uploads/docs/VSA Bitgood.pdf

[18] Goodwin, M.S., Velicer, W.F. and Intille, S.S. (2008). Telemetric monitoring in the behavior sciences. Behavior Research Methods, 40 (1), 328-341.

[19] MACLin, O.H., MACLin, M.K., Peterson, D., Chowdhry, O. and Priyanka, J. (2009). Social psychophysics to answer "social" questions with PsychoPro. Behavior Research Methods, 41 (3), 623-632.

[20] Leech, N.L. and Onwuegbuzie, A.J. (2009). A typology of mixed methods research designs. Quality \& Quantity: International Journal of Methodology, 43, pp. 265-275.

[21] Onwuegbuzie, A.J., Johnson, R.B. and Collins, K.M.T. (2011). Assessing legitimation in mixed research: a new framework. Quality \& Quantity: International Journal of Methodology, 45, pp. 1253-1271. 
Table 1. Relative temperatures on face and hands for two visitor cases

\begin{tabular}{|c|c|c|c|c|c|c|c|c|c|}
\hline \multicolumn{5}{|c|}{ Sequence A - Male Visitor } & \multicolumn{5}{|c|}{ Sequence B - Female Visitor } \\
\hline Photo & Topic \& Sequence & $T_{m}^{* *}$ & Face $^{*}$ & Hand* & Photo & Topic \& Sequence & $T_{m}^{* *}$ & Face* & Hand* \\
\hline P1M & Plasma Ball, during visit & 25.10 & 9.30 & 9.24 & P1F & Text Panel, during visit & 26.50 & 7.40 & 6.10 \\
\hline P2M & Plasma Ball, during visit & 25.00 & 9.40 & 9.34 & $\mathrm{P} 2 \mathrm{~F}$ & Iter, during visit & 28.30 & 6.50 & 4.48 \\
\hline P3M & Plasma Ball, during visit & 25.70 & 9.50 & 8.90 & P3F & Iter, during visit & 28.00 & 6.90 & 4.60 \\
\hline P4M & Iter, during visit & 25.30 & 10.40 & 9.60 & P4F & Iter, during visit & 28.60 & 6.30 & 4.50 \\
\hline P5M & Iter, during visit & 26.60 & 9.30 & 8.20 & P5F & Reactor site, during visit & 27.30 & 7.60 & 6.40 \\
\hline P6M & Reactor site, during visit & 26.90 & 8.60 & 7.44 & P6F & Text Panel, during visit & 27.20 & 7.70 & 5.60 \\
\hline P7M & Reactor site, during visit & 26.10 & 9.30 & 8.70 & P7F & Bicycle, beginning to pedal & 26.00 & 8.00 & 6.00 \\
\hline P8M & Text Panel, during visit & 26.40 & 9.30 & 7.94 & P8F & Bicycle, continuing to pedal & 26.10 & 7.20 & 5.80 \\
\hline P9M & Text Panel, during visit & 26.50 & 9.10 & 7.84 & P9F & Bicycle, during visit & 26.00 & 7.80 & 5.30 \\
\hline P10M & Bicycle, beginning to pedal & 24.50 & 10.60 & 10.10 & P10F & Assessment, after visit & 27.10 & 7.60 & 6.50 \\
\hline P11M & Bicycle, continuing to pedal & 24.50 & 10.70 & 9.80 & P11F & Assessment, after visit & 27.10 & 7.50 & 7.20 \\
\hline P12M & Assessment, after visit & 27.90 & 7.20 & 5.40 & P12F & Assessment, after visit & 26.80 & 7.70 & 6.30 \\
\hline P13M & Assessment, after visit & 28.30 & 6.60 & 5.10 & P13F & Plasma Ball, after visit & 25.50 & 7.60 & 6.90 \\
\hline Mean & & 26.06 & 9.18 & 8.28 & Mean & & 26.96 & 7.37 & 5.82 \\
\hline
\end{tabular}

(*) Relative Temperatures $\left(T_{r}\right)$ in degrees Celsius $\left({ }^{\circ} \mathrm{C}\right)$

$\left({ }^{\star \star}\right)$ Average Absolute Environment Temperatures in degrees Celsius $\left({ }^{\circ} \mathrm{C}\right)$

${ }^{(* *)}$ Relative Temperature Function $\left(T_{r}\right)=T_{a}-T_{m}$

Table 2. Average relative temperatures and correlates of face and hands for two visitor cases

\begin{tabular}{|l|c|c|c|c|}
\hline & Face $^{*}$ & Hand $^{*}$ & Face - Hand $^{\star \star}$ & (Face/Hand) $^{\star \star \star}$ \\
\hline Male Visitor (13) & 9.18 & 8.28 & 0.90 & 0.96 \\
\hline Female Visitor (13) & 7.37 & 5.82 & 1.55 & 0.71 \\
\hline Male + Female (26) & 8.27 & 7.05 & 1.22 & 0.95 \\
\hline Diff. (Male - Female) & 1.81 & 2.46 & -0.65 & \\
\hline
\end{tabular}

$\left({ }^{*}\right)$ Relative Temperatures in degrees Celsius $\left({ }^{\circ} \mathrm{C}\right)$

$\left({ }^{\star *}\right)$ Differential Temperatures in degrees Celsius $\left({ }^{\circ} \mathrm{C}\right)$

$\left.{ }^{\star \star \star}\right)$ Pearson's Correlation 
Table 3. Descriptive characteristics of thermography and thermal correlates throughout the guided tour

\begin{tabular}{|l|l|c|c|c|c|c|c|}
\hline & & \multicolumn{2}{|c|}{ Male Visitor } & \multicolumn{2}{|c|}{ Female Visitor } & \multicolumn{2}{c|}{ Average (Male + Female) } \\
\hline \multirow{2}{*}{ Exhibition } & $\begin{array}{l}\text { Category } \\
\text { (Frequency) }\end{array}$ & Face* & Hands & Face & Hands* & Face* & Hands $^{*}$ \\
\hline \multirow{5}{*}{ Topic } & Plasma Ball (4) & 9.40 & 9.16 & 7.60 & 6.90 & 8.95 & 8.60 \\
\cline { 2 - 8 } & Iter Model (5) & 9.85 & 8.90 & 6.57 & 4.53 & 7.88 & 6.28 \\
\cline { 2 - 8 } & $\begin{array}{l}\text { Reactor Site Model } \\
\text { (3) }\end{array}$ & 8.95 & 8.07 & 7.60 & 6.40 & 8.50 & 7.51 \\
\cline { 2 - 8 } & Text Panels (4) & 9.20 & 7.89 & 7.55 & 5.85 & 8.38 & 6.87 \\
\cline { 2 - 8 } & Bicycle (5) & 10.65 & 9.95 & 7.67 & 5.70 & 8.86 & 7.40 \\
\cline { 2 - 8 } & Assessment (5) & 6.90 & 5.26 & 7.60 & 6.67 & 7.32 & 6.10 \\
\hline \multirow{5}{*}{ Time } & During (20) & 9.59 & 8.83 & 7.27 & 5.42 & 8.55 & 7.29 \\
\cline { 2 - 8 } & After (6) & 6.90 & 5.26 & 7.60 & 6.73 & 7.37 & 6.23 \\
\hline \multirow{5}{*}{ Participation } & Active (9) & 8.78 & 7.60 & 7.58 & 6.48 & 8.11 & 6.98 \\
\cline { 2 - 7 } & Passive (17) & 9.36 & 8.58 & 7.24 & 5.41 & 8.36 & 7.09 \\
\hline
\end{tabular}

$\left.{ }^{*}\right)$ Relative Temperatures in degrees Celsius $\left({ }^{\circ} \mathrm{C}\right)$

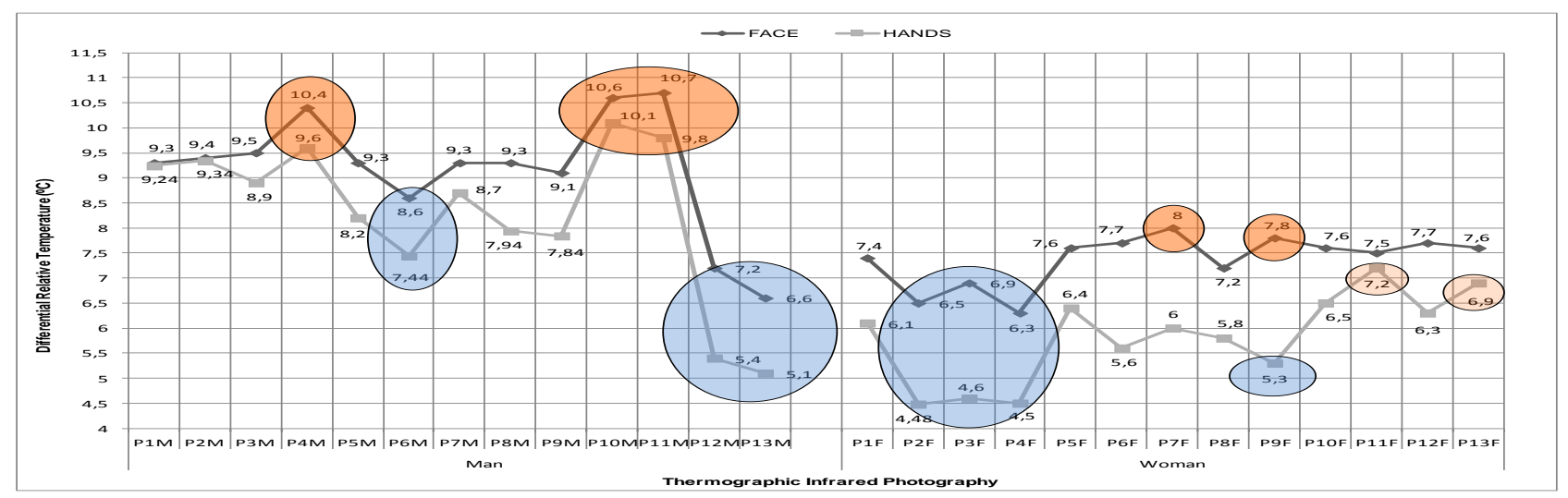

Fig. 1. Thermal variations across museum visitor tasks

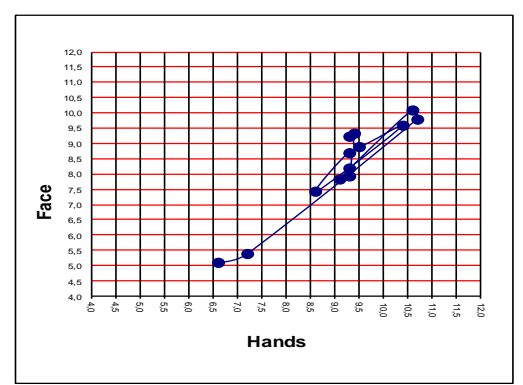

Fig. 2. Face and hand scattergram for the male subject $(r=0.96)$

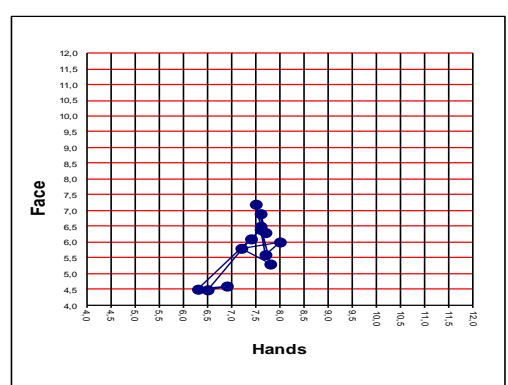

Fig. 3. Face and hand scattergram for the female subject $(r=0.71)$

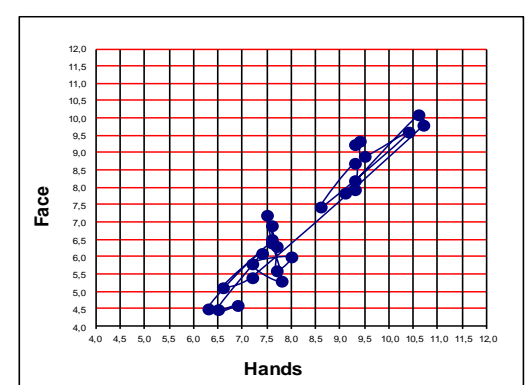

Fig. 4. Face and hand scattergram for males and females ( $r=0.95)$ 


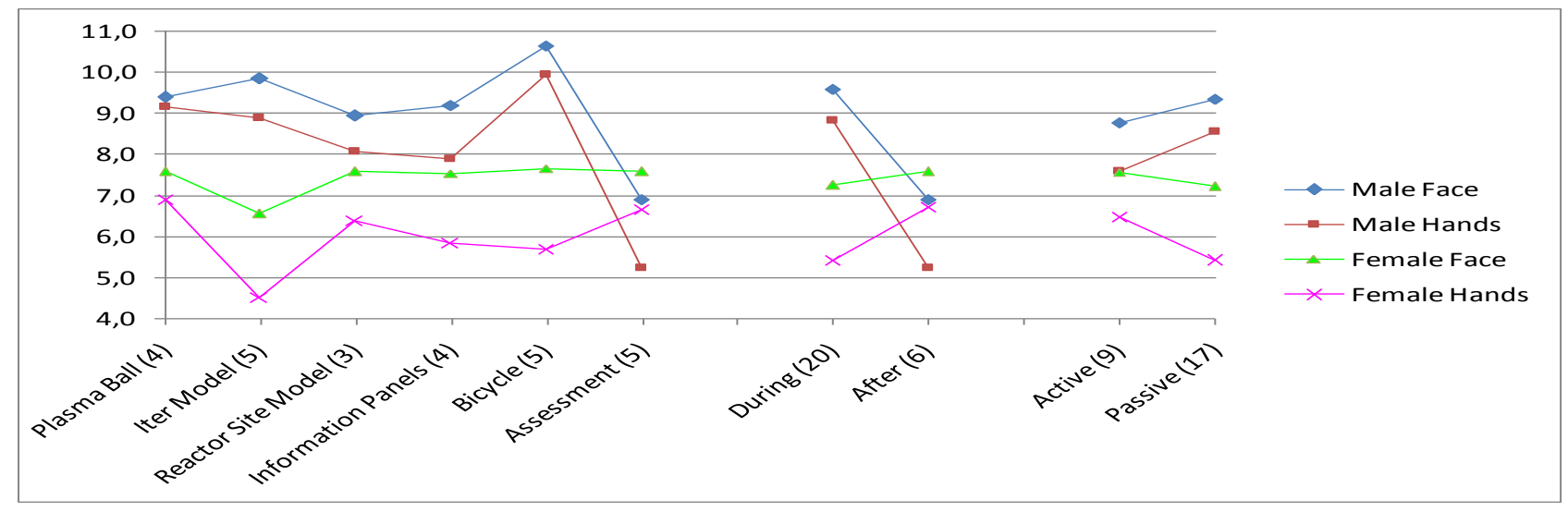

Fig. 5. Line chart of thermography descriptive characteristics for the male and female subjects

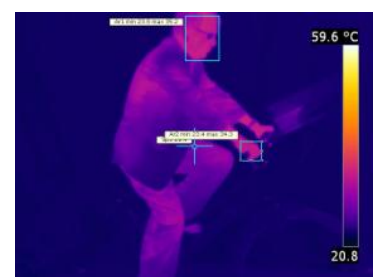

Fig. 6. (P11M). Male visitor. DRMATF $\left(10.7^{\circ} \mathrm{C}\right)$ in an active participation role.

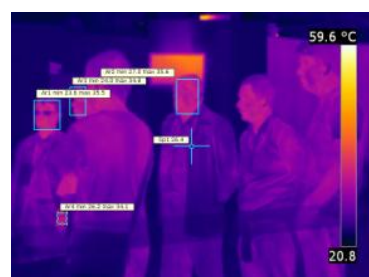

Fig. 7. (P6M). Male visitor. DRMITF $\left(8.6^{\circ} \mathrm{C}\right)$ in an observer role.

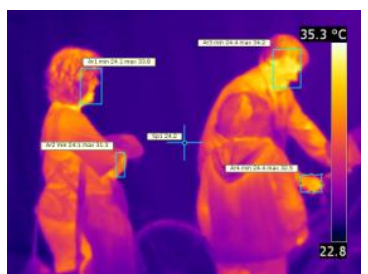

Fig. 8. (P9F). Female visitor. DRMATF $\left(7.8^{\circ} \mathrm{C}\right)$ in a passive participation role.

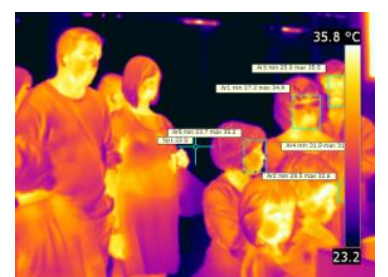

Fig.9. (P3F). Female visitor. DRMITF $\left(6.9^{\circ} \mathrm{C}\right)$ in an observer role. 